\title{
The design of foldable chair based on omnidirectional moving with rescuable platform
}

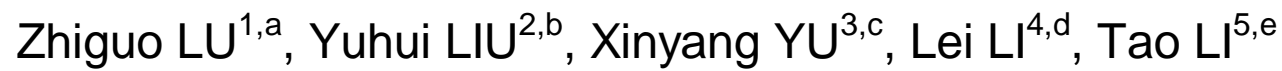 \\ 1,2,3,4,5 Department of Mechanical Engineering and Automation, Northeastern University, Shenyang \\ 110004, China \\ azglu@me.neu.edu.cn, 'laiterliu@163.com, clingyu2468@163.com, ${ }^{d} 1003198249 @ q q . c o m$, \\ e18304048653@163.com
}

Keywords: molding; transferring goods; manned patrol; electromagnetic clutch

Abstract: Under the Solidworks design environment, the modeling of folding chair based on the omnidirectional mobile rescue platform has been finished. This integral structure design can achieve two functions of transshipment goods and manned patrol, and the electromagnetic clutch is used to control the chair reversing. The optimum designing has been realized by analyzing the static mechanical properties of some important components under the ANSYS environment. This folding chair is compact and flexible and is suitable for various emergency rescues.

\section{Introduction}

Many kinds of damage happened in worldwide annually and those disasters can cause a large area of buildings collapsed and casualties. According to the time of happening, disaster relief can be divided into three stages - before the disaster, disaster relief and post-disaster relief [1]. But the complexity of the relief phase, risk and urgency brought great difficulty to rescue work. After the disaster, the most urgent thing is to transport relief supplies and survivors and research suggests that if these survivors cannot get effective relief within 48 hours, mortality rate would increase sharply [2]. In general, a large number of rescue equipment and supplies can be carried through traditional rescue transportation only, but the rescue vehicle is large generally and cannot reach disaster sites. Rescuer have no choice but to walk the most road through with Rescue equipment and supplies, which reduced efficiency greatly. Therefore, in order to improve the efficiency of rescue and transport efficiency, looking for a small vehicle, using as a rescue vehicle, especially in the upper body with a mobile and flexible structure that makes up for the inadequacy of traditional rescue vehicle material stack space, it is necessary to assist rescuer who transport equipment, supplies and wounded.

\section{Contents}

This design is a folding chairs based on omnidirectional mobile rescue platform. Under the Solidworks environment, we have designed, completed the motion simulation analysis, realized the virtual design process [3], simulated some important parts by ANSYS and produced finally. Taking the idea of double modular design with which we complete the design of the folding chair. One of the module is the seat module, the other is the electromagnetic clutch module. The function is different from another [4]. Connecting the two modules together by screw, which formed folding chairs. The seat stay on the omnidirectional mobile rescue vehicle and 2 electromagnetic clutch were used between the bottom of the seat and omnidirectional mobile platform, keeping a horizontal freedom degree. When we need a different transportation function, the seat of the state can be changed with it that the seat can collapse or expand, thus it can realize the piled material or check in the injured and normal patrol two functions respectively. Assembling the folding chair with the existing omnidirectional mobile platform, it can be used as a new kind of rescue vehicles which help rescuer to transport the equipment, supplies and wounded. That improve the efficiency of rescue and transporting efficiency, and it is good in simple structure, operating easily, flexible moving and adaptable. 


\section{Assembly design}

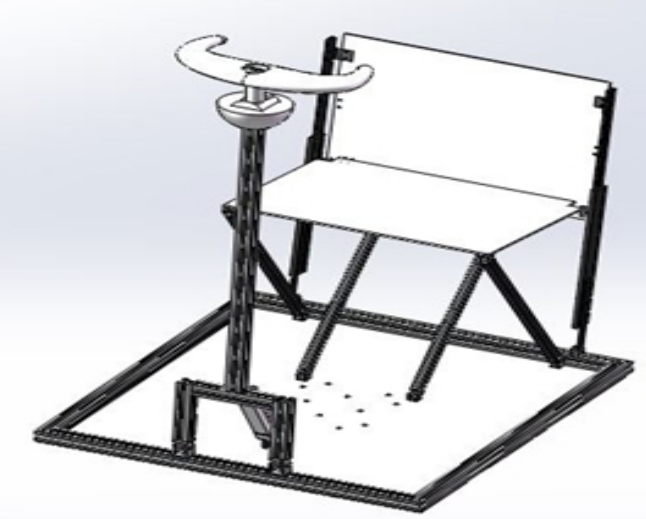

Fig. 1. The assembly diagram of expanded seat module

As shown in Fig. 1, the module of folding chair include seat plate, back plate and 4 legs that two of them is fixed length and others can be folded into half. Folding point is connected by hinge in half of legs and there is installed a lock catch which can control folding leg's state (bend or unbend) through opening and closing the lock catch. In addition, a zero bar is added between 2 folding legs and prevent bracing piece from load and deformation. 4 legs are hinge joint between seat plate and pedestal respectively. With the same time, fixing length legs and folding legs are crossed setting in the space. Back plate is located in back of seat plate and both sides are installed a sliding rail with chute, the bottom of sliding rail is spliced on the pedestal and the back plate slide by relative move between sliding and chute.

Handrail modules contains handrail and bracing piece. Handrail located in top of bracing piece that is spliced on pedestal, with a control panel. A linear actuator is installed between pedestal and bracing piece and has an impact on bracing piece's inclination angle through operating. Electromotive handspike is a displacement implementing and position control mechanism [5]. And two main function of it are constructing a triangle with bracing piece and pedestal and adapting different height of patrol when they are cruising with a comfortable posture.

\section{Function}

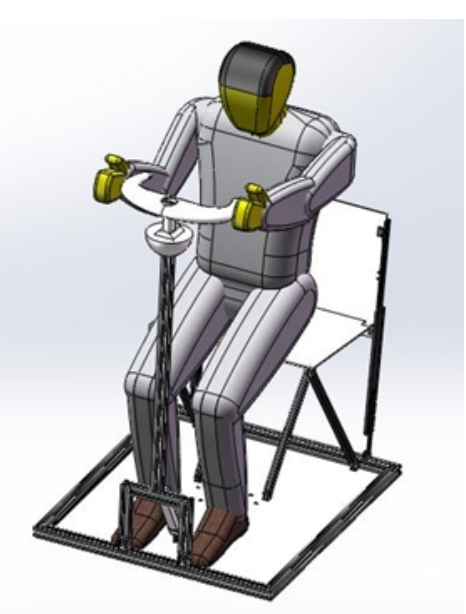

Fig. 2. Expanded schematic

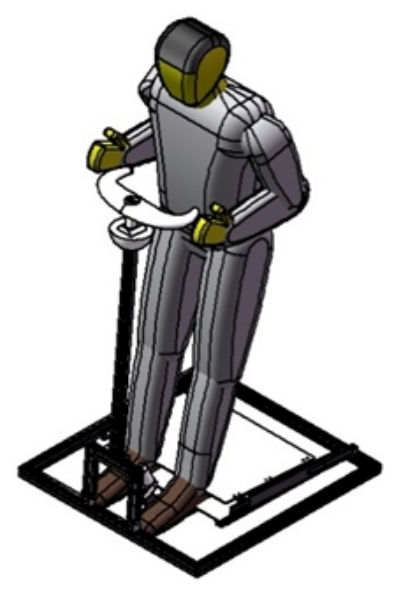

Fig. 3. Closed schematic

\section{Manned}

When the folding chair is opened, as shown in Fig. 2, navigating mate set down and drive the car. In order to improve labor efficiency and keep the most comfortable posture which is suitable for human 
engineering for navigating mate in driving and balance the difference on body and height between drivers, we can adjust the stretching and drawing of linear actuator through control panel [6]. And then, the bracing piece has been adjust to perfect angle.

\section{Loading}

When someone wants to pack the folding chair up, as shown in Fig. 3, the first step is opening the lock catch. Then, pushing the seat plate down until the seat plate abutt pedestal with the falling fixed legs. After that, we push the back plate and make it sliding through chute until the lowest state even in level. Another surface can be used as working stage. For example, drivers are standing on stage and driving with rescue equipment or goods.

\section{Electromagnetic clutch module}

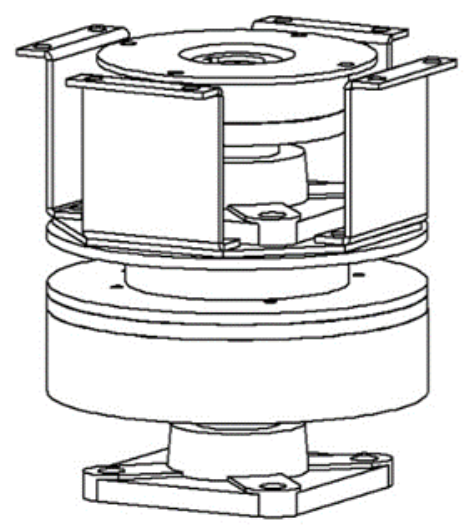

Fig. 4. The assembly diagram of electromagnetic clutch module

Electromagnetic clutch also called electromagnetic coupling, as shown in Fig. 4, using electromagnetic energy, and moment of force is transferred from master end to slave end, connect mechanical structure and delivered machine driven system function [7]. Although the omnidirectional mobile rescue platform have a powerful maneuverable capability and can suitable for complex road condition, we need control the state of electromagnetic clutch depends on reality condition, energize or power failure, which determine the direction of advance.

The bottom of this module is fixed on the platform through bearing support and top of that is fixed under the central of pedestal with a bearing support and 4 fabrications. A shaft is used to connect 2 clutches in case partiality and a needle bearing is placed between 2 clutches. The needle bearing is small but possess a strong bearing capacity [8]. When you need to keep the folding chair relative rest, we connect the electromagnetic clutch's power. And then, the horizontal rotational degree of freedom between platform and pedestal is limited; when you need to keep the folding chair rotation, we interrupt the electromagnetic clutch's power. At the same time, the chair can be carried out with platform in a horizontal rotation. 


\section{Statics analysis in ANSYS}

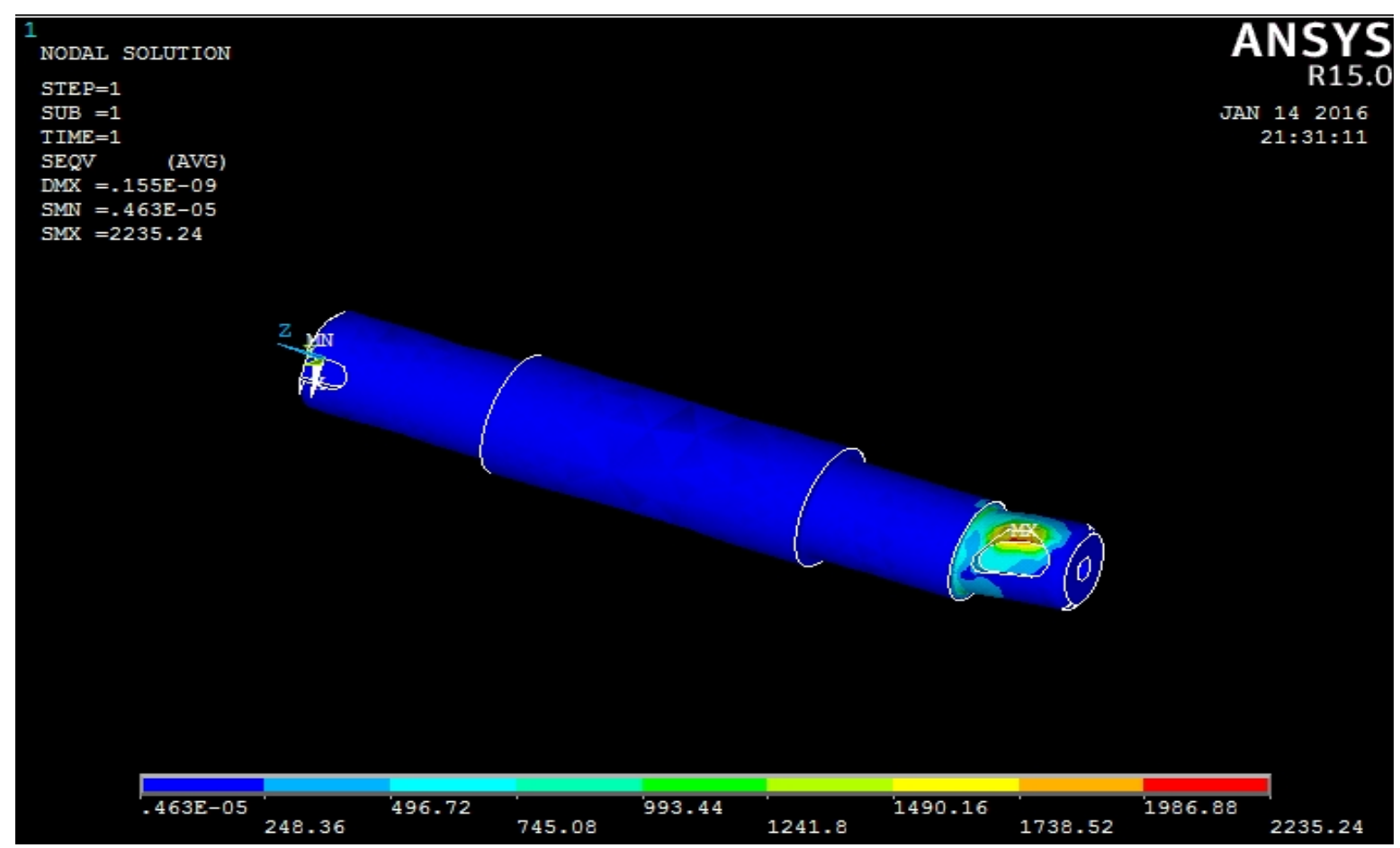

Fig. 5. Static analyzing with shaft in ANSYS

By adding a fictitious loading on shaft, we analyze its statics characteristic in ANSYS and find that the main force area is in keyway during using. The key, usually is used to connect shaft and components which installed on shaft, fixed circumference and transmit torque [9]. As Fig. 5 illustrated, two sides of keyway afford mainly shearing force. We may increase the allowable specific pressure or adapt tangent key connection rather than enlarge the bore of electromagnetic clutch that is standard parts in optimization design. Besides, as middle seize of shaft is small, we could also shrink diameter to reduce raw material with lower cost.

\section{Conclusions}

Under the Solidworks design environment, we have modeled the chair and analyzed interference, which increase its feasibility in theory; in order to provide a good report for this design, we also used ANASY to analyze important components loading and numerical simulation [10]. The foldable chair based on omnidirectional moving with rescuable platform can suitable well in narrow space, especially in complex cases, and make up for temporarily rescuable platform.

\section{Acknowledgement}

This project is supported by 9\#Students Innovation and Entrepreneurship Training Program (No.150049). National Natural Science Foundation of China (51505069), Fundamental Research Funds for the Central Universities (N140304008), the Research Fund for the Doctoral Program of Higher Education of China (20130042120027), Science and Technology Planning Project of Liaoning Province(2015020150), Science and Technology Planning Project of Shenyang City(F15-123-9-00). The authors would like to take their gratitude to the Innovation and Entrepreneurship College of Northeastern University, China, for its supporting.

\section{References}

[1] Jinguo Liu. Current research, key performances and future development of search and rescue robot $[\mathrm{J}]$. Chinese journal of mechanical engineering, 2006, 42(12):1-11. 
[2] Bing Li. The research of snake-like robot and the application in disaster rescue [N]. Robot Technique and Application, 2003, 3: 22-26.

[3] Yufeng Luo. Parametric feature modeling technology research based on the Solidworks[J]. Journal of machine design, 2004, 2(21): 52-54.

[4] Zhisen Zhang. All-in-One Integration Design of Folding Desk and Chair Based on CATIA [N]. Mechanical Engineer, 2015, 4: 156-157.

[5] Mina Jia. Practical electromotive handspike [N]. Mechanical management and development, 2006, 2:58-59.

[6] $\mathrm{Yu}$ X. Study on based motion in improved radon transformation with mechanics properties [J]. Advanced Materials Research, 2013, 703:300-303.

[7] Fei Zhao. The Design Optimization and Dynamic Simulation on High-torque an Electromagnetic Clutch [D]. Harbin: Harbin Engineering University, 2011.

[8] Guoxing Ma. ANSYS-enabled finite element analysis on needle bearings [N]. Chinese journal of mechanical engineering, 2008, 6(3):328-332.

[9] Zhili Sun et al. Mechanical design [M]. Beijing: Science Press, 2008.

[10]Ziqiang Zhang. Static Analysis of Multi-Function Chair Based onANSYS[N]. Mechanical Engineer, 2015,5:15-16. 